\title{
Plasma Brain Natriuretic Peptide Level After Hybrid Therapy With Pulmonary Vein Isolation and Antiarrhythmic Drugs for Atrial Fibrillation
}

\author{
Takumi Yamada, ${ }^{1}$ MD, Yoshimasa MuraKami, ${ }^{2} \mathrm{MD}$, Taro OKADA, ${ }^{2} \mathrm{MD}$, \\ Naoki YoshIDA, ${ }^{2}$ MD, Junji ToYAMA, ${ }^{2}$ MD, Yukihiko YoshIDA, ${ }^{3}$ MD, \\ Naoya TsuboI, ${ }^{3} \mathrm{MD}$, Yasuya INDEN, ${ }^{4} \mathrm{MD}$, Makoto HIRAI, ${ }^{4} \mathrm{MD}$, \\ and Toyoaki MuroHARA, ${ }^{4} \mathrm{MD}$
}

\section{SUMMARY}

The atrial fibrillation (AF) recurrence rate after pulmonary vein isolation (PVI) has been relatively high and in some unsuccessful PVI cases, antiarrhythmic drugs that were ineffective before PVI may become effective (hybrid therapy). The purpose of this study was to investigate the relationship between the brain natriuretic peptide (BNP) level and the effect of the hybrid therapy.

In 28 lone AF patients undergoing hybrid therapy, the plasma BNP level was measured before PVI and before and 3 months after administering an antiarrhythmic drug. Fifteen patients were free of AF after the hybrid therapy (effective group), and 13 still had recurrent AF after (noneffective group). At baseline, in all patients the BNP level was elevated, and there were no significant differences in the BNP level between the 2 groups. The BNP level was significantly decreased after PVI in the effective group $(56.8 \pm 23.1$ versus $37.5 \pm 16.7 \mathrm{pg} / \mathrm{mL}, P<0.05)$ but not in the noneffective group $(74.3 \pm 47.8$ versus $79.7 \pm 54.4 \mathrm{pg} / \mathrm{mL}, \mathrm{NS})$. The elevated BNP level normalized in all effective group patients. The criterion consisting of a net value of $<60.0 \mathrm{pg} / \mathrm{mL}$ or a reduction in the BNP level of $>10.0 \mathrm{pg} / \mathrm{mL}$ after PVI predicted the effective group patients with a high accuracy.

A significant reduction in the BNP level after PVI may be a useful predictor of the responders to antiarrhythmic drug therapy in patients with recurrent AF after PVI. The hybrid therapy may be effective in patients whose PVs serve as a dominant AF substrate. (Int Heart J 2008; 49: 143-151)

Key words: Atrial fibrillation, Brain natriuretic peptide, Pulmonary vein isolation, Hybrid therapy, Radiofrequency catheter ablation

From the ${ }^{1}$ Division of Cardiovascular Disease, University of Alabama at Birmingham, Alabama, USA, ${ }^{2}$ Division of Cardiology, Aichi Prefectural Cardiovascular and Respiratory Center, ${ }^{3}$ Division of Cardiology, Nagoya Dai-ni Red Cross Hospital, Cardiovascular Center, and ${ }^{4}$ Department of Cardiology, Nagoya University Graduate School of Medicine, Aichi, Japan.

Address for correspondence: Takumi Yamada, MD, Division of Cardiovascular Disease, Cardiac Rhythm Management Laboratory, University of Alabama at Birmingham, VH B147, 1670 University Boulevard, 1530 3rd AVE S, Birmingham, AL 35294-0019, USA.

Received for publication November 19, 2007.

Revised and accepted February 14, 2008. 
IT is known that during atrial fibrillation (AF), an inappropriately rapid ventricular response, loss of the atrial contribution to the cardiac output, and atrial overloading, lead to the activation of the neurohormonal system. ${ }^{1)}$ It has been demonstrated that successful elimination of AF by pulmonary vein isolation (PVI) normalizes the elevated plasma brain natriuretic peptide (BNP) level. ${ }^{2-4)}$ Though PVI has been established as a reliable therapy for curing AF, the recurrence rate has been relatively high and a repeat ablation procedure is sometimes required. ${ }^{5-8)}$ It has been reported that in some cases of unsuccessful ablation, antiarrhythmic drugs that were ineffective before the PVI may become effective (hybrid therapy) after. ${ }^{5,9,10)}$ However, the relationship between the BNP level and the effect of the hybrid therapy remains unknown. The purpose of this study was to investigate the relationship.

\section{METHODS}

Patient characteristics: The study population was selected from 96 consecutive symptomatic AF patients ( 80 men, $59 \pm 10$ years), 65 with paroxysmal AF (PAF), 17 with persistent AF (Pers-AF), and 14 with permanent AF (Perm-AF) who were refractory to $3 \pm 1$ class I or class III antiarrhythmic drugs (not including amiodarone). The AF type was defined according to the international consensus. ${ }^{11)}$ AF patients who were administered class II or class IV antiarrhythmic drugs before and after PVI were not enrolled because of the potential of those drugs to modify the results of the study by directly or indirectly affecting the autonomic nerve system. The mean duration of $\mathrm{AF}$ was $4 \pm 3$ years (1 to 18). The mean echocardiographic dimension of the left atrium (LA) was $35 \pm 5 \mathrm{~mm}$ (25 $\mathrm{mm}$ to $45 \mathrm{~mm}$ ) and mean left ventricular (LV) ejection fraction $67 \pm 8 \%$ (55\% to $90 \%$ ). For the Doppler echocardiographic parameters, the mean E/A wave ratio was $0.9 \pm 0.3$ ( 0.4 to 1.7$)$ and the mean deceleration time was $198 \pm 19 \mathrm{~ms}(160$ $\mathrm{ms}$ to $235 \mathrm{~ms}$ ). No patient had any structural heart disease, LV diastolic dysfunction for their age, or renal dysfunction. Angiotensin-converting enzyme inhibitors were administered in 4 patients and angiotensin II type 1 receptor blockers in 9 patients due to hypertension. The Institutional Review Board approved the study protocol, and all patients provided written informed consent. All antiarrhythmic drugs were discontinued for at least 5 half-lives prior to the study.

Electrophysiological study and PVI procedure: Catheterization into the LA was performed with a one puncture and two-sheath technique. Intravenous heparin was administered to maintain an activated clotting time $>250$ seconds after the atrial transseptal procedure. In all cases, circumferential PVI targeting the PV antrum potentials of 3 to $4 \mathrm{PVs}$ was performed using a $31 \mathrm{~mm}$ multielectrode basket catheter (MBC) (Constellation ${ }^{\mathrm{TM}}$, EP Technologies, Boston Scientific Corpo- 
ration, San Jose, CA, USA) as previously reported. ${ }^{4)}$ Radiofrequency energy was delivered with a target temperature of $55^{\circ} \mathrm{C}$ and maximum power output of $40 \mathrm{~W}$ for 60 seconds (EPT-1000TC generator, EP Technologies), using an 8-mm tip catheter (Blazer II 5770T, EP Technologies). Successful PVI was defined as either the abolition or dissociation of the distal PV potentials. Extensive LA ablation was never added after the PVI. When spontaneous AF was observed even after the PVI, focal ablation targeting the non-PV AF foci was also performed.

Administration of antiarrhythmic drugs: During the follow-up period, no antiarrthythmic drugs were administered to any of the patients at first. When AF recurred after the PVIs, only one class I or class III antiarrhythmic drug that failed to control the AF before the first procedure was administered.

Blood sampling and hormone assay: Before the PVIs and before and 3 months after administration of the antiarrhythmic drugs, blood samples to measure the BNP level were obtained from the antecubital vein in the supine position after a resting period of 30 minutes immediately before an echocardiographic examination. If the latest paroxysmal AF attack persisted for more than 8 hours, blood samples were obtained at least 3 hours after the paroxysmal AF terminated. The BNP level was determined using a chemi-luminescent enzyme immunoassay (CLEIA).

Assessment of AF attacks: All of the patients were contacted and asked if they had had any attacks concerning their rhythm problem before the PVI and before and 3 months after administration of the antiarrhythmic drug. Twenty-four hour Holter monitoring was also performed at least twice before the ablation procedure to evaluate the reliability of the patients' answers.

Follow-up: Clinical follow-up was performed at 2 weeks, 1 month and every month thereafter, using 24-hour Holter and cardiac recordings in all patients. All patients who reported symptoms were given an event monitor to document the cause of the symptoms. No patients were lost to follow-up.

Statistical analysis: Continuous variables are expressed as the group mean \pm 1 $\mathrm{SD}$. Comparisons of the continuous variables between the groups were analyzed using Student's $t$-test or ANOVA, as appropriate. The categorical variables expressed as numbers and percentages were compared using the chi-square test. Statistical significance was selected as a value of $P<0.05$.

\section{RESULTS}

PV isolation, hybrid therapy and follow-up: Successful isolation of 3 to 4 PVs could be achieved in all patients. At more than 3 months of follow-up after the first PVI, AF recurred without any antiarrhythmic drugs in 14 (22\%) PAF, 13 (76\%) Pers-AF, and 11 (79\%) Perm-AF patients. Twenty-one (6 PAF, 8 Pers-AF, 
and 7 Perm-AF) of those patients with recurrent AF after the first PVI underwent a second session. Because the electrophysiological study revealed the recurrence of electrical connections between the LA and one to two PVs previously ablated in all of these patients, the PVI was repeated using the same technique as in the first session. In 13 of these patients, focal ablation targeting non-PV AF foci was also performed. Ten patients (2 PAF, 5 Pers-AF, and 3 Perm-AF) were free of symptomatic AF without any antiarrhythmic drugs after the second procedure. Eventually, a total of 68 patients were free of symptomatic AF without any antiarrhythmic drugs after multiple catheter ablation procedures (ablation group). In the remaining 28 patients with recurrent $\mathrm{AF}$ after one or two procedures, 8 (5 PAF, 2 Pers-AF, and 1 Perm-AF) after the first procedure and 7 (5 PAF, 1 Pers$\mathrm{AF}$, and 1 Perm-AF) after the second procedure became free of symptomatic AF after the administration of one antiarrhythmic drug (cibenzoline in 5 PAF, 3 PersAF, and 2 Perm-AF, flecainide in 3 PAF, and bepridil in 2 PAF) that failed to control the AF before the procedure (effective group). The remaining 13 patients still had recurrent AF after the hybrid therapy (noneffective group). Eight (4 PAF, 3 Pers-AF, and 1 Perm-AF) of these patients continued to be followed-up because both the frequency and duration of the AF attacks had decreased and consequently the symptoms had shown remarkable improvement. The remaining 5

Table. Clinical Characteristics

\begin{tabular}{lccc}
\hline & $\begin{array}{c}\text { Effective } \\
(n=15)\end{array}$ & $\begin{array}{c}\text { Noneffective } \\
(n=13)\end{array}$ & $P$ \\
\hline Age (years) & $58 \pm 12$ & $53 \pm 7$ & $\mathrm{NS}$ \\
Gender (male/female) & $13 / 2$ & $11 / 2$ & $\mathrm{NS}$ \\
AF duration (years) & $5 \pm 4$ & $6 \pm 6$ & $\mathrm{NS}$ \\
Paroxysmal/Persistent/Permanent AF & $10 / 3 / 2$ & $4 / 3 / 6$ & $\mathrm{NS}$ \\
Echocardiographic parameters & & & \\
$\quad$ LA dimension & $36 \pm 5$ & $36 \pm 5$ & $\mathrm{NS}$ \\
$\quad$ LVEF & $65 \pm 8$ & $65 \pm 13$ & $\mathrm{NS}$ \\
Catheter ablation & $3.9 \pm 0.3$ & $3.8 \pm 0.4$ & $\mathrm{NS}$ \\
$\quad$ Electrically isolated PVs (n/cases) & $1.4 \pm 0.5$ & $1.3 \pm 0.5$ & $\mathrm{NS}$ \\
$\quad$ No. of procedures & $2(13 \%)$ & $2(15 \%)$ & $\mathrm{NS}$ \\
$\quad$ No. of patients with non-PV foci successfully ablated & $6(40 \%)$ & $3(23 \%)$ & $\mathrm{NS}$ \\
$\quad$ Cavo-tricuspid isthmus ablation & & & \\
Medication & $1(7 \%)$ & $0(0 \%)$ & $\mathrm{NS}$ \\
$\quad$ ACE-Is & $3(20 \%)$ & $2(15 \%)$ & $\mathrm{NS}$ \\
$\quad \begin{array}{l}\text { ARBs } \\
\text { Antiarrhythmic drugs } \\
\text { (cibenzoline/flecainide/bepridil) }\end{array}$ & $10 / 3 / 2$ & $7 / 4 / 2$ & $\mathrm{NS}$ \\
\hline
\end{tabular}

ACE-I indicates angiotensin-converting enzyme inhibitor; AF, atrial fibrillation; ARB, angiotensin II receptor blocker; LA, left atrial; LVEF, left ventricular ejection fraction; No., number; and PV, pulmonary vein. 


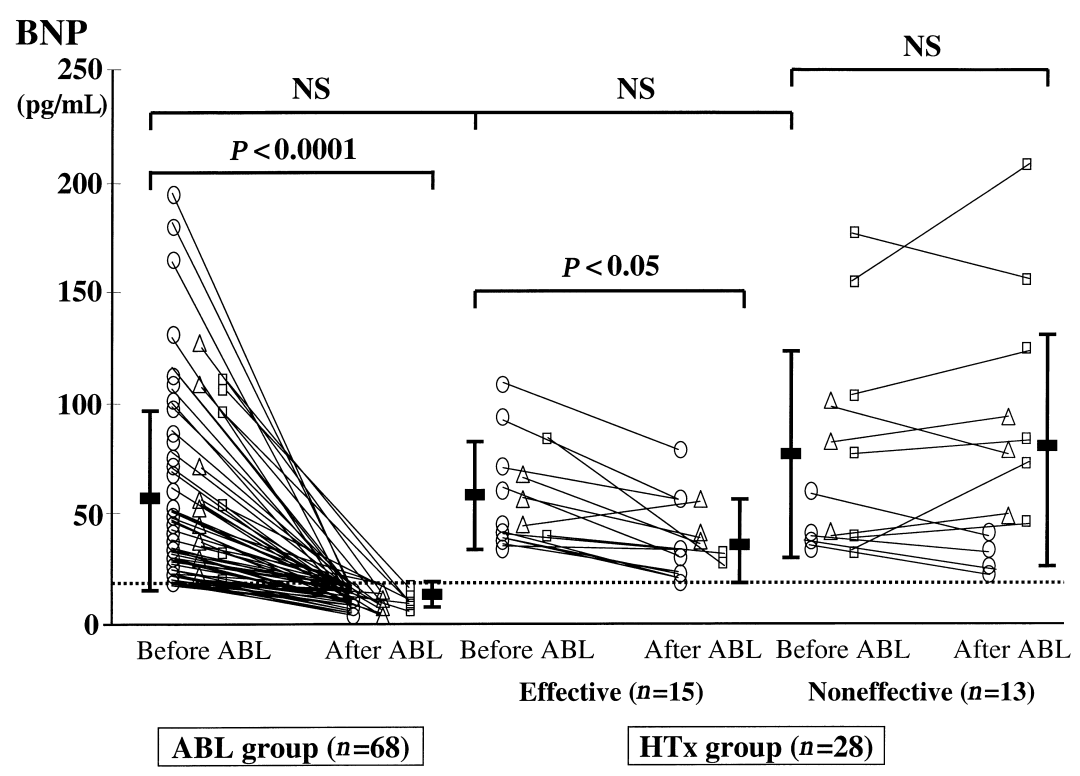

Figure 1. Change in plasma brain natriuretic peptide (BNP) level in atrial fibrillation (AF) patients who underwent multiple catheter ablation procedures (ABL).

The circles indicate paroxysmal AF patients, triangles persistent $\mathrm{AF}$ patients, squares permanent $\mathrm{AF}$ patients, and dotted line the normal limit of the plasma BNP level. HTx indicates hybrid therapy.

patients with recurrent Perm-AF continued to be followed-up with medication aiming at a rate-control.

Comparison of the clinical and electrophysiological parameters between the effective and noneffective group patients: The results of the clinical and electrophysiological parameters in the patients who underwent the hybrid therapy are shown in the Table. There were no significant differences in any of the clinical and electrophysiological parameters such as age, male gender, AF duration, AF type, echocardiographic parameters, number of electrically isolated PVs, number of PVI procedures, number of patients with non-PV foci successfully ablated, proportion of a cavo-tricuspid isthmus ablation which was performed before the PVI, and medications, between the effective and noneffective group patients.

Plasma BNP level: At baseline, the BNP level was elevated in all patients in this study and there were no significant differences among the ablation group patients and the effective and noneffective group patients who underwent the hybrid therapy $(55.9 \pm 41.5$ versus $56.8 \pm 23.1$ versus $74.3 \pm 47.8, P=0.32)$ (Figure 1$)$. The elevated BNP level at baseline normalized in all the ablation group patients (55.9 \pm 41.5 versus $12.3 \pm 4.4 \mathrm{pg} / \mathrm{mL}, P<0.0001$ ), whereas it did not decrease to within normal limits in any of the patients who underwent the hybrid therapy because of 


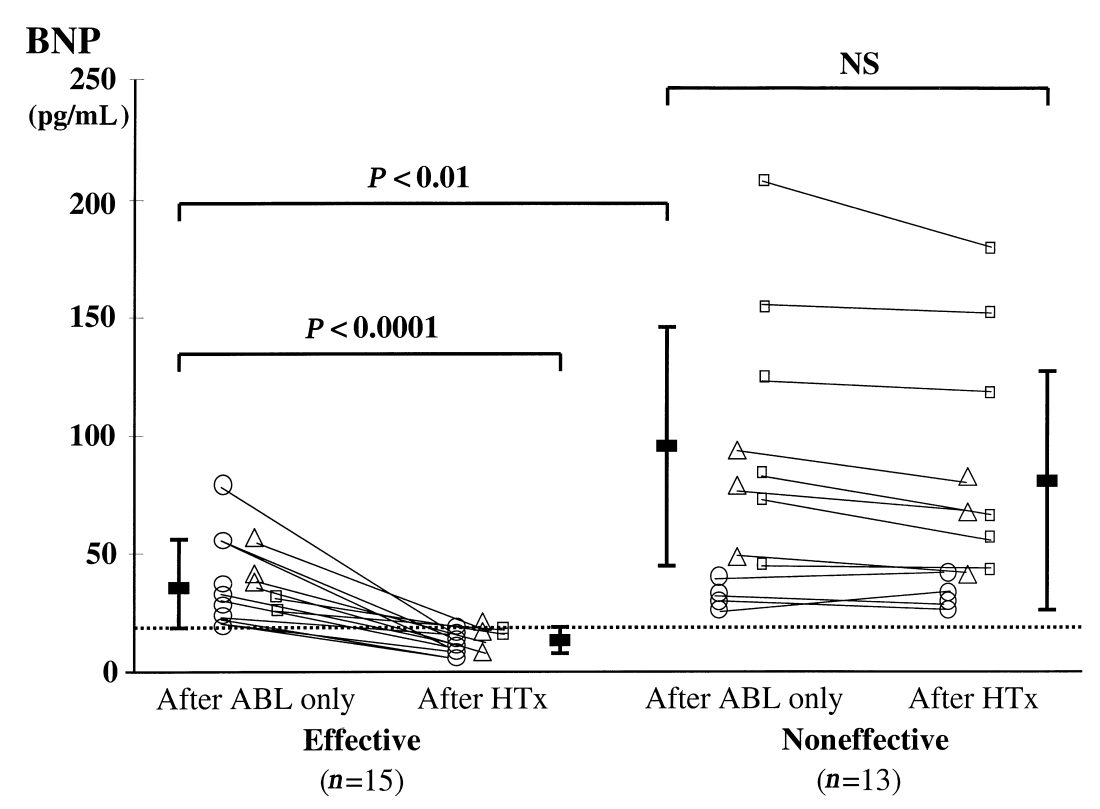

Figure 2. Plasma BNP level before and after the administration of one antiarrhythmic drug. The abbreviations are the same as in Figure 1.

$\mathrm{AF}$ recurrence after multiple catheter ablation procedures (Figure 1). In the effective group patients, the BNP level decreased significantly after the PVI procedures $(56.8 \pm 23.1$ versus $37.5 \pm 16.7 \mathrm{pg} / \mathrm{mL}, P<0.05)$, but did not in the noneffective group patients $(74.3 \pm 47.8$ versus $79.7 \pm 54.4 \mathrm{pg} / \mathrm{mL}$, NS) (Figure 1). The BNP level before the administration of the antiarrhythmic drugs was significantly greater in the noneffective group patients than in the effective group patients $(79.7 \pm 54.4$ versus $37.5 \pm 16.7, P<0.01)$ (Figure 2). In the effective group patients, the elevated BNP level at baseline significantly decreased to within normal limits after administration of the antiarrhythmic drugs (37.5 \pm 16.7 versus $12.8 \pm 4.1 \mathrm{pg} / \mathrm{mL}, P<0.0001)$, but did not decrease significantly in the noneffective group patients $(94.8 \pm 52.8$ versus $72.2 \pm 48.9 \mathrm{pg} / \mathrm{mL}$, NS) (Figure 2). The criterion that the BNP level after PVI $<60.0 \mathrm{pg} / \mathrm{mL}$ predicted effective group patients was associated with a sensitivity of $93.3 \%$, specificity of $53.8 \%$, positive predictive accuracy of $70.0 \%$, and negative predictive accuracy of $87.5 \%$ (Figure 3). The criterion that the reduction of BNP level after PVI $>10.0 \mathrm{pg} / \mathrm{mL}$ predicted effective group patients was associated with a sensitivity of $86.7 \%$, specificity of $83.3 \%$, positive predictive accuracy of $81.2 \%$, and negative predictive accuracy of $83.3 \%$ (Figure 3). 

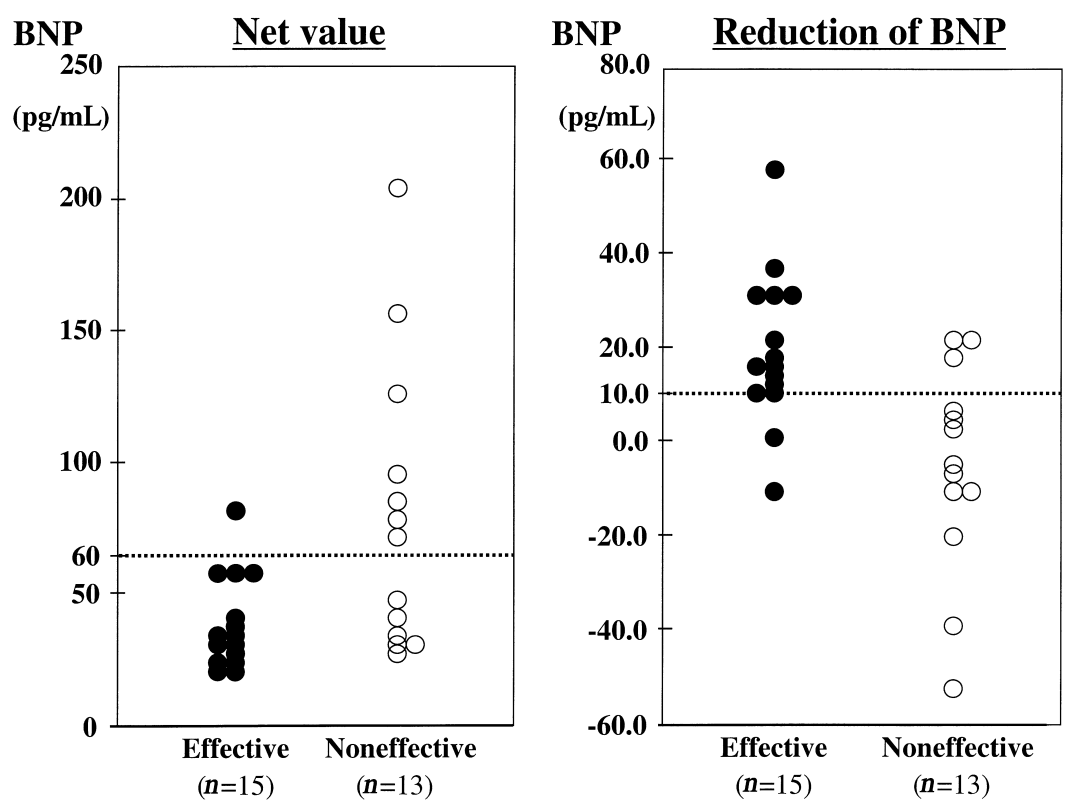

Figure 3. The net value and reduction in the plasma BNP level after the catheter ablation procedures. The abbreviations are the same as in Figure 1.

\section{Discussion}

In some cases, an attempt to cure AF, especially persistent and permanent AF, by PVI alone may be challenging. ${ }^{5,6,9,10)}$ This study demonstrated that elevated BNP levels normalized in the patients with AF which was refractory to antiarrhythmic drugs and PVI when the AF was eliminated by the hybrid therapy with PVI followed by the administration of one antiarrhythmic drug. These findings suggest that in consideration of the neurohormonal system, hybrid therapy may be an effective therapeutic approach with which to cure recurrent AF after PVI. Previous studies have demonstrated that the BNP level can be a useful marker for evaluating the efficacy of PVI in AF patients. ${ }^{2-4)}$ This study also demonstrated that the BNP level could be a useful marker for evaluating the efficacy of antiarrhythmic drug therapy in patients with recurrent AF after PVI.

Over the last decade, considerable advances have been made in the catheter ablation of AF. The success rates have improved with the evolution and development of PVI. ${ }^{12,13)}$ However, the risk of critical complications such as LA flutter $^{13,14)}$ and atrio-esophageal fistula ${ }^{15)}$ remains significant. Therefore, hybrid therapy may be considered as an alternative to repeated PVI. In this study, the BNP level before PVI was not helpful for predicting the efficacy of the hybrid 
therapy. However, the criterion of the net value $<60.0 \mathrm{pg} / \mathrm{mL}$ or reduction $>10.0$ $\mathrm{pg} / \mathrm{mL}$ in BNP level after PVI predicted effective group patients with high accuracy. When a significant reduction in the BNP level is observed despite AF recurrence after PVI, the strategy of the following therapy may be selected considering that the patients may respond well to hybrid therapy.

Tojo, et al speculated 2 reasons for explaining why hybrid therapy could eliminate AF which was refractory to antiarrhythmic drugs and PVI. ${ }^{9)}$ First, the creation of pharmacologic block in partially isolated PVs may contribute to the hybrid effect. Second, antiarrhythmic drugs may not be able to suppress focal discharges from PVs, but may be able to suppress those in atrial tissue (non-PV foci). However, the relationship between the dominance of the AF substrates, PVs or non-PV foci, and the efficacy of the hybrid therapy remains unclear. In this study, the change in the BNP level after the PVI demonstrated that PVI was effective in the effective group patients with the hybrid therapy, but was not effective in the noneffective group patients. These findings suggest that the hybrid therapy might be effective in patients whose PVs served as a dominant AF substrate.

Study limitations: It is known that the antiarrhythmic drugs administered after PVI in this study have multiple electrophysiological effects including Ca channel blocking effects, ${ }^{16,17)}$ which might have affected the BNP level. However, we believe that the Ca channel blocking effect on the BNP level was not sufficiently large to change the results of this study because the antiarrhythmic drugs were used similarly in both the effective and noneffective group patients.

Recent reports using transtelephonic and long-term Holter monitoring have revealed that asymptomatic AF recurrences after an AF ablation occur more often than we would expect, and thus freedom from AF after PVI may be overestimated. ${ }^{18,19)}$ In this study, asymptomatic AF recurrences might have been missed and the cure rate might have been a little overestimated because intermittent Holter recordings alone were performed for the clinical follow-up. Further studies using continuous 7-day Holter monitoring as used in previous reports ${ }^{18,19)}$ will be needed to investigate whether BNP is useful for evaluating asymptomatic AF recurrences after PVI.

Conclusions: Successful hybrid therapy with PVI followed by the administration of one antiarrhythmic drug normalized the elevated BNP level in patients with AF which was refractory to antiarrhythmic drugs and PVI. The significant reduction in the BNP level after PVI may be a useful predictor of responders to antiarrhythmic drug therapy in patients with recurrent AF after PVI. Hybrid therapy may be effective in patients whose PVs serve as a dominant AF substrate. 


\section{REFERENCES}

1. Roy D, Paillard F, Cassidy D, et al. Atrial natriuretic factor during atrial fibrillation and supraventricular tachycardia. J Am Coll Cardiol 1987; 9: 509-14.

2. Date T, Yamane T, Inada K, et al. Plasma brain natriuretic peptide concentrations in patients undergoing pulmonary vein isolation. Heart 2006; 92: 1623-7.

3. Yamada T, Murakami Y, Okada T, et al. Plasma atrial natriuretic peptide and brain natriuretic peptide levels after radiofrequency catheter ablation of atrial fibrillation. Am J Cardiol 2006; 97: 1741-4.

4. Kurosaki K, Tada H, Hashimoto T, et al. Plasma natriuretic peptide concentrations as a predictor for successful catheter ablation in patients with drug-refractory atrial fibrillation. Circ J 2007; 71: 313-20.

5. Haïssaguerre M, Shah DC, Jaïs $\mathrm{P}$, et al. Electrophysiological breakthroughs from the left atrium to the pulmonary veins. Circulation 2000; 102: 2463-5.

6. Oral H, Knight BP, Tada H, et al. Pulmonary vein isolation for paroxysmal and persistent atrial fibrillation. Circulation 2002; 105: 1077-81.

7. Yamada T, Murakami Y, Okada T, et al. Can segmental pulmonary vein ablation reduce the recurrence of atrial fibrillation when using a higher RF power, larger tip electrode catheter, and additional RF deliveries?: the limitations of point-by-point RF ablation. Int Heart J 2006; 47: 219-28.

8. Ohkubo K, Watanabe I, Okumura Y, et al. Pulmonary vein isolation for atrial fibrillation in patients with paroxysmal atrial fibrillation and prolonged sinus pause. Int Heart J 2007; 48: 247-52.

9. Tojo H, Kumagai $\mathrm{K}$, Noguchi $\mathrm{H}$, et al. Hybrid therapy with pilsicainide and pulmonary vein isolation for atrial fibrillation. Circ J 2005; 69: 1503-7.

10. Yamada T, Murakami Y, Okada T, et al. Electrophysiological pulmonary vein antrum isolation with a multielectrode basket catheter is feasible and effective for curing paroxysmal atrial fibrillation: efficacy of minimally extensive pulmonary vein isolation. Heart Rhythm 2006; 3: 377-84.

11. Lévy S, Camm AJ, Saksena S, et al; Working Group on Arrhythmias of European Society of Cardiology; Working Group of Cardiac Pacing of European Society of Cardiology; North American Society of Pacing and Electrophysiology. International consensus on nomenclature and classification of atrial fibrillation: A collaborative project of the Working Group on Arrhythmias and the Working Group of Cardiac Pacing of the European Society of Cardiology and the North American Society of Pacing and Electrophysiology. J Cardiovasc Electrophysiol 2003; 14: 443-5.

12. Oral H, Scharf C, Chugh A, et al. Catheter ablation for paroxysmal atrial fibrillation: Segmental pulmonary vein ostial ablation versus left atrial ablation. Circulation 2003; 108: 2355-60.

13. Ouyang F, Bänsch D, Ernst S, et al. Complete isolation of left atrium surrounding the pulmonary veins: new insights from the double-Lasso technique in paroxysmal atrial fibrillation. Circulation 2004; 110: 2090-6.

14. Mesas CE, Pappone C, Lang CC, et al. Left atrial tachycardia after circumferential pulmonary vein ablation for atrial fibrillation: electroanatomic characterization and treatment. J Am Coll Cardiol 2004; 44: 1071-9.

15. Pappone C, Oral H, Santinelli V, et al. Atrio-esophageal fistula as a complication of percutaneous transcatheter ablation of atrial fibrillation. Circulation 2004; 109: 2724-6.

16. Kodama I, Ogawa S, Inoue H, et al. Profiles of aprindine, cibenzoline, pilsicainide and pirmenol in the framework of the Sicilian Gambit. The Guideline Committee for Clinical Use of Antiarrhythmic Drugs in Japan (Working Group of Arrhythmias of the Japanese Society of Electrocardiology). Jpn Circ J 1999; 63: 1-12. (Review)

17. Izumi D, Chinushi M, Watanabe $\mathrm{H}$, et al. Bepridil for drug-refractory ventricular tachyarrhythmias. Intern Med 2007; 46: 119-24.

18. Karch MR, Zrenner B, Deisenhofer I, et al. Freedom from atrial tachyarrhythmias after catheter ablation of atrial fibrillation: a randomized comparison between 2 current ablation strategies. Circulation 2005; 111: 2875 80.

19. Piorkowski C, Kottkamp H, Tanner H, et al. Value of different follow-up strategies to assess the efficacy of circumferential pulmonary vein ablation for the curative treatment of atrial fibrillation. J Cardiovasc Electrophysiol 2005; 16: 1286-92. 